Hershkovitz - Montiaceae taxonomy

\title{
Taxonomy of Montiaceae: critical clarifications and corrections
}

\author{
Mark A. Hershkovitz \\ Santiago, Chile \\ cistanthe@gmail.com
}

\begin{abstract}
This work provides critical commentary on and corrections of a recently published taxonomy of Montiaceae. Valid citation and publication dates of novel taxa per the International Code of Nomenclature for Plants, Algae, and Fungi are summarized.

KEY WORDS: Montiaceae, taxonomy, nomenclature.
\end{abstract}

\section{Introduction}

Hershkovitz (2019a) published a new subfamilial taxonomy of Montiaceae that validated the names of 24 new taxa. Multiple earlier circulated versions of this work (Hershkovitz, 2018a, 2019b, and versions thereof) included most of these and a few other nomenclatural novelties, but these did not constitute valid publication per the International Code of Nomenclature for Plants, Algae, and Fungi (Shenzhen Code; Turland et al., 2018), with particular reference to Article 30.2. Accordingly, Hershkovitz (2019a) and no other facsimile should be referenced for the nomenclature contained therein. Other or differently parsed taxon names in different versions distributed otherwise are not valid. For convenience and clarification, the validated names and publication dates in Hershkovitz (2019a) are summarized below. Discretionally holo-/lectotypified names are indicated with an asterisk. Additional lectotypifications in Hershkovitz (2019a) are superfluous, because typification follows from the taxon name. I report here that the name Calyptridinae Hershk. is superfluous, and Calandrinia Kunth sect. Caespitosa Phil. was typified erroneously.

\section{Suprageneric taxon names validated in Hershkovitz (2019a)}

The suprageneric taxonomy in Hershkovitz (2019a) is "incomplete" in that only reasonably wellevidenced clades of two or more genera were named. Corresponding higher taxa of remaining singleton genera were not named, nor were named higher taxa not well corroborated by phylogenetic evidence. Although suprageneric taxonomic ranks were not designated explicitly, they were deliberately implicit in grammatical suffixes that correspond with those specified in the Code (Turland et al., 2018). The newly validated suprageneric taxa are:

Cistantheae Hershk., Phytoneuron 2019-27: 54. 6 May 2019.

CalYPTRIDINAE Hershk., Phytoneuron 2019-27: 59. 6 May 2019, nom. superfl. $\equiv$ Calyptridiinae Franz, Bot. Jahrb. 42 Beibl. 97: 44. $1908 \equiv$ Calyptrideae (Franz) McNeill, Taxon 23: 727. 1974.

Phemerantheae Hershk., Phytoneuron 2019-27: 64. 6 May 2019. This taxon was named with the wrong suffix. An earlier draft of Hershkovitz (2019a) raised Montioideae to effectively subfamilial rank, but failed to revise the suffix of Phemerantheae accordingly. Although Phemerantheae Hershk. remains valid, in context, it should have been called Phemeranthoideae. 
Hershkovitz - Montiaceae taxonomy

Generic name validated in Hershkovitz (2019a)

*THINGIA Hershk., Phytoneuron 2019-27: 61. 6 May 2019. LECTOTYPE: Thingia ambigua (S. Watson) Hershk. $\equiv$ Claytonia ambigua S. Watson $\equiv$ Calandrinia ambigua (S. Watson) Howell $\equiv$ Cistanthe ambigua (S. Watson) Carolin ex Hershk.

\section{Subgeneric names validated in Hershkovitz (2019a)}

*Cistanthe Spach sect. Rosulatae (Reiche) Hershk., Phytoneuron 2019-27: 56. 6 May 2019 Calandrinia sect. Rosulatae Reiche, Ber. Deutsch. Bot. Ges. 15: 502. 1897. LECTOTYPE: Cistanthe longiscapa (Barnéoud) Carolin ex Hershk. $\equiv$ Calandrinia longiscapa Barnéoud.

*Cistanthe sect. Rosulatae subsect. Thyrsoideae Hershk., Phytoneuron 2019-27: 58. 6 May 2019. LECTOTYPE: Cistanthe thyrsoidea (Reiche) Peralta and D.I. Ford $\equiv$ Calandrinia thyrsoidea Reiche.

*CALYPTRIDIUM sect. SPRAgUEA (Torr.) Hershk., Phytoneuron 2019-27: 61. 6 May $2019 \equiv$ Spraguea Torr., Smithsonian Contr. Knowl. 6: 4. 1853. LECTOTYPE: Calyptridium monospermum Greene $\equiv$ Spraguea monosperma (Greene) Torr. $\equiv$ Cistanthe monosperma (Greene) Hershk.

\section{Binomials validated in Hershkovitz (2019a)}

CALYPTRIDIUM Hessae (J. T. Thomas) Hershk., Phytoneuron 2019-27: 60. 6 May 2019 $\equiv$ Calyptridium parryi A. Gray var. hessae J.T. Thomas, Leafl. W. Bot. 8: 10. $1956 \equiv$ Cistanthe parryi (A. Gray) Hershk. var. hessae (J.T. Thomas) Kartesz \& Ghandi, Phytologia 71: 62. 1991.

CalyPTRIDIUM MarTiRenSE (Guilliams, M. G. Simpson \& Rebman) Hershk., Phytoneuron 201927: 61. 6 May $2019 \equiv$ Calyptridium parryi A. Gray var. martirense Guilliams, M.G. Simpson \& Rebman, Madroño 58: 260. 2012 [2011].

Calyptridium nevadense (J. T. Howell) Hershk., Phytoneuron 2019-27: 61. 6 May 2019 $\equiv$ Calyptridium parryi A. Gray var. nevadense J.T. Howell, Leafl. W. Bot. 4: 216. $1945 \equiv$ Calyptridium parryi A. Gray subsp. nevadense (J.T. Howell) Munz, Fl. S. Calif. 711. $1974 \equiv$ Cistanthe parryi (A. Gray) Hershk. var. nevadense Kartesz \& Ghandi, Phytologia 71: 62. 1991.

*Cistanthe Aegitalis (F. Phil.) Carolin ex Hershk., Phytoneuron 2019-27: 55. 6 May 2019 $\equiv$ Calandrinia aegitalis F. Phil., Anales Univ. Chile 85: 184. 1893. LECTOTYPE: F. Philippi s.n. (SGO).

*Cistanthe Chamissoi (Barnéoud) Carolin ex Hershk., Phytoneuron 2019-27: 57. 6 May 2019 $\equiv$ Calandrinia chamissoi Barnéoud in Gay, Fl. Chil. 2(4): 497-498. 1846 [1847] E Calandrinia arenaria Cham. var. chamissoi Reiche, Fl. Chile 2: 346.1898 and An. Univ. Chile 100: 350. 1898. LECTOTYPE: Bertero 1348, (G: G440496), ISOTYPES: G: G440495, G440494; P: P01903300, SYNTYPE: Bertero 683 (P! P01903301; NOTE: Bertero 683 evidently is a mixed collection that includes the type of Talinum trigonum Colla). 
*Cistanthe Crassifolia (Phil.) Carolin ex Hershk., Phytoneuron 2019-27: 56. 6 May 2019 $\equiv$ Calandrinia crassifolia Phil., Anales Univ. Chile 85: 180. 1893. LECTOTYPE: F. Philippi s.n. (SGO).

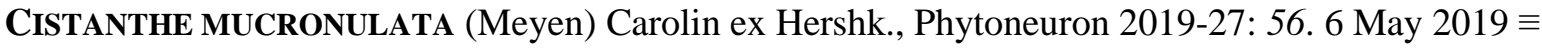
Calandrinia mucronulata Meyen, Reise Erde 1: 314. 1834. TYPE: not located.

*Cistanthe Oblongifolia (Barnéoud) Carolin ex Hershk., Phytoneuron 2019-27: 57. 6 May 2019 三Calandrinia oblongifolia Barnéoud in Gay, Fl. Chil. 2(4): 482(-483). 1846 [1847]. LECTOTYPE: Gay 316 (P: P019033038).

*CistanTHe SUbSPECIOSA Hershk., Phytoneuron 2019-27: 56. 6 May 2019. LECTOTYPE: Werdermann 405 (E: E:00033178), ISOTYPES: F, U, US.

Cistanthe TRIGONA (Colla) Hershk., Phytoneuron 2019-27: 57. 6 May 2019 = Talinum trigonum Colla, Mem. Realle Accad. Torino 37: 71. 1834. LECTOTYPE: not designated, ISOTYPES: Bertero 683 (G, G00440471, G00440472, G00440473, G00440474; mixed collections that include syntypes of Calandrinia chamissoi Barnéoud).

*Cistanthe Vicina (Phil.) Carolin ex Hershk., Phytoneuron 2019-27: 58. 6 May 2019 $\equiv$ Calandrinia vicina Phil., Anales Univ. Chile 85: $301.1893 \equiv$ Calandrinia arenaria Cham. var. vicina (Phil.) Reiche, Fl. Chile 2: 346.1898 and An. Univ. Chile 100: 350. 1898. LECTOTYPE: Philippi s.n. (SGO).

PhilipPiamra ARANCiOANA (Peralta) Hershk., Phytoneuron 2019-27: 63. 6 May 2019 三 Cistanthe arancioana Peralta, Gayana Bot. 52: 45. 1995.

PhilipPiamra CalyCina (Phil.) Hershk., Phytoneuron 2019-27: 63. 6 May 2019 Calandrinia calycina Phil., Fl. Atacam. 21. $1860 \equiv$ Cistanthe calycina (Phil.) Carolin ex Hershk., Phytologia 70: 220. 1991.

PhiliPPIAMra Densiflora (Barnéoud) Hershk., Phytoneuron 2019-27: 63. 6 May 2019 $\equiv$ Calandrinia densiflora Barnéoud in Gay, Fl. Chil. 2(4): 503. 1846 [1847] 三 Cistanthe densiflora (Barnéoud) Carolin ex Hershk., Phytologia 70: 220. 1991.

PhilipPiamra MinusCula (Cullen) Hershk., Phytoneuron 2019-27: 63. 6 May 2019 三 Calandrinia minuscula Cullen, Bol. Soc. Arg. Bot. 5: 12. 1953 ECistanthe minuscula (Cullen) Peralta in Kiesling, Fl. San Juan 1: 163. 1994.

PhiliPPIAMra SAlsoloides (Barnéoud) Hershk., Phytoneuron 2019-27: 63. 6 May $2019 \equiv$ Calandrinia salsoloides Barnéoud in Gay, Fl. Chil. 2(4): 502(-503). 1846 [1847] 三 Cistanthe salsoloides (Barnéoud) Carolin ex Hershk. in Phytologia 68: 269. 1990.

*Thingia AMBIGUA (S. Watson) Hershk., Phytoneuron 2019-27: 61. 6 May 2019 三 Claytonia ambigua S. Watson, Proc. Amer. Acad. Arts 17: 365(-366). $1882 \equiv$ Calandrinia ambigua (S. Watson) Howell, Erythea 1: 34.1893 三 Cistanthe ambigua (S. Watson) Carolin ex Hershk., Phytologia 68: 269. 1990. 
Hershkovitz - Montiaceae taxonomy

\section{Additional Montiaceae names contemporaneously validated elsewhere}

*Cistanthe FLORESIORUM J. M. Watson., Int. Rock Gardener 111: “25” [pagination absent; page determined excluding journal cover page] . March 2019. HOLOTYPE: A. R. Flores \& J. M. Watson 11750 (SGO); ISOTYPES: CONC, herb. Flores \& Watson.

*Cistanthe Philhershkovitztiana Hershk., Phytologia 100: 209. 21 December 2018. HOLOTYPE: Hershkovitz 19-01 (F), ISOTYPE: (P).

\section{Other noteworthy nomenclatural observations/corrections}

In addition to the above novelties, Hershkovitz' (2019a) taxonomic revision included a few longoverlooked synonyms. These include Tutuca Molina (= Calandrinia Kunth), T. chilensis Molina (?= Calandrinia compressa Schrad.), and T. fistulosa (nom. superfl., = T. chilensis Molina). Three sections of Calandrinia were recognized as having been validly published: $C a$. sect. Amarantoidea Phil., $C a$. sect. Caespitosae Phil., and Ca. sect. Grandiflorae Phil. The first two of these have priority over the later synonyms, respectively, $\mathrm{Ca}$. sect. Amarantoideae Reiche and $\mathrm{Ca}$. sect. Acaules Reiche. These later synonyms are superfluous, because they refer exactly to the sections as circumscribed and named by Philippi. Hershkovitz (2019a and earlier versions) failed to correct the typification of $C a$. sect. Caesapitosae. Per Article 10.8 (Turland et al., 2018), it must be Calandrinia caespitosa Gillies ex Arn. Calandrinia sect. Grandiflorae has priority in Calandrinia over $C a$. sect. Cistanthe Reiche, not only because of historical precedent, but because of Art. 10.8 (Turland et al., 2018), since this section includes Calandrinia grandiflora Lindl. [三 Cistanthe grandiflora (Lindl.) Schltdl., nom. conserv.]. In the genus Cistanthe, the section is named Cistanthe sect. Cistanthe.

\section{Discussion}

The taxonomy of Montiaceae elaborated in Hershkovitz (2019a) originated in 2017 as a rather skeletal table or appendix in manuscripts focusing on Montiaceae natural history (e.g., evolution, ecology, geography), as well as theory. At that time, no satisfactory current and cohesive taxonomy existed spanning the supraspecific up to the familial level. As the preliminary effort elaborated in length and complexity, it was spun off from the more theoretical work (Hershkovitz, 2018b, c) into a separate work with an intentionally "scientistic" title (Hershkovitz, 2018a). That title reflects its initial rejection in the biological sciences preprint archive bioRxiv.org. A new logically constructed taxonomic analysis was not considered per se to embody a new scientific analysis. Further research and refinement yielded multiple versions of Hershkovitz (2019b), but, per Article 30.2 (Turland et al., 2018), taxonomic novelties were not validated prior to Hershkovitz (2019a). As demonstrated here, Hershkovitz (2019a) includes some "bugs." These errors bring great shame to my village. Nonetheless, I am so confident that the taxonomy will facilitate new Montiaceae research that I guarantee that if consumers are not completely satisfied, the complete purchase price will be refunded cheerfully.

\section{Acknowledgements}

Elaboration of the present paper would not have been possible except for the generous support and dedicated efforts of Katia Chechelnitzky, Sandra Steuermann, and Gabriela Feldman of Fundación Reshet in Chile (www.reshet.cl). 
Hershkovitz - Montiaceae taxonomy

\section{Literature Cited}

Hershkovitz, M. [A.] 2018a. Synopsis of a new taxonomic synthesis of Montiaceae (Portulacineae) based on rational metadata analysis, with critical new insights on historically poorly understood taxa and a reevaluation of historical biogeography. Preprints 2018080496. https://doi.org/10.20944/preprints201808.0496.v2

Hershkovitz, M.[A.] 2018b. Perspectives from Montiaceae (Portulacineae) evolution. I. Phylogeny and phylogeography. Preprints 2018090096. https://doi.org/10.20944/preprints 201809.0096. v2

Hershkovitz, M.[A.] 2018c. Perspectives from Montiaceae (Portulacineae) evolution. II. Ecological evolution, phylogenetic comparative analysis, and the principle of evolutionary idiosyncraticity. Preprints 2018090566. https://doi.org/10.20944/preprints201809.0566.v2

Hershkovitz, M. A. 2019. Systematics, Evolution, and Phylogeography of Montiaceae (Portulacineae). Phytoneuron 2019-27: 1-77. http://www.phytoneuron.net/2019Phytoneuron/27PhytoNMontiaceae.pdf

Hershkovitz, M. [A.]. 2019b. Systematics, Evolution, and Phylogeography of Montiaceae (Portulacineae). Preprints 201903. https://doi.org/10.20944/preprints201903.0206.v32

Turland, N. J., J. H. Wiersema, F. R. Barrie, W. Greuter, D. L. Hawksworth, P. S. Herendeen, S. Knapp, W.-H. Kusber, D.-Z Li, K. Marhold, T. W. May, J. McNeill, A. M. Monro, J. Prado, M. J. Price, and G. F. Smith (eds.). 2018. International Code of Nomenclature for Algae, Fungi, and Plants (Shenzhen Code) adopted by the Nineteenth International Botanical Congress Shenzhen, China, July 2017. Regnum Vegetabile 159. Koeltz Botanical Book, Glashütten.

https://doi.org/10.12705/Code.2018 